\title{
DNA condensation and redissolution: Interaction between overcharged DNA molecules.
}

\author{
E.Allahyarov ${ }^{1,2,3}$, G.Gompper ${ }^{2}$, H.Löwen ${ }^{1}$ \\ 1 Institut für Theoretische Physik II, Heinrich-Heine-Universität \\ Düsseldorf, D-40225 Düsseldorf, Germany \\ 2 Institut für Festkörperforschung, Forschungszentrum Jülich, D-52425 Jülich, Germany \\ 3 Institute for High Temperatures, Russian Academy of Sciences, Izhorskaya 13/19, 117419 \\ Moscow, Russia
}

(February 26, 2019)

\begin{abstract}
The effective DNA-DNA interaction force is calculated by computer simulations with explicit tetravalent counterions and monovalent salt. For overcharged DNA molecules, the interaction force shows a double-minimum structure. The positions and depths of these minima are regulated by the counterion density in the bulk. Using two-dimensional lattice sum and free energy perturbation theories, the coexisting phases for DNA bundles are calculated. A DNA-condensation and redissolution transition and a stable mesocrystal with an intermediate lattice constant for high counterion concentration are obtained.
\end{abstract}

PACS: 87.15.Kg, 61.20Ja, 82.70.Dd, 87.10+e

Typeset using REVTEX 


\section{INTRODUCTION}

Long DNA is naturally found in a dense form in most biological systems due to the presence of compacting agents. In vitro the most used agents are polyamines such as trivalent spermidine (Spd) and tetravalent spermine (Spe) [1]. These agents play a key role in maintaining cellular DNA in a compact state [2,3], modulate ion channel activities of cells, are essential for normal cell growth [4] and can effectively be applied in gene delivery and in the field of genetic therapy. Under physiologic ionic and $\mathrm{pH}$ conditions, the polyamines are positively charged and hence DNA is their prime target of interaction. The molecular mechanism of polyamine function in DNA condensation is presumed to involve neutralization of the negatively charged DNA backbone by the positively charged amino groups of Spd and Spe [5]. Experimental results and counterion condensation theories indicate that non-specific interactions are predominantly electrostatic between polyamines and DNA phosphates [6-9]. Thus, the electrostatic shielding of phosphates facilitates close helix-helix surface contacts and, ultimately, DNA condensation through the correlation attraction [10], the attraction between strongly correlated counterion layers on the adjacent DNA surfaces.

In the last decade, different experiments $[3,11-16]$ have shown evidence of DNA redissolution (i.e. DNA unbinding) with increasing concentration of polyamines and several of their structural analogues. First, addition of a certain amount of multivalent salt causes the collapse of single DNA or the bundling in multi-columnar structures. Upon addition of more salt, the polyelectrolyte redissolves and DNA assumes again an unbundled conformation. There are three experimentally well established features of the redissolution phenomena: i) a linear relationship is found between the threshold concentration of multivalent ions $C_{c}$, when the onset of DNA condensation takes place, and the initial DNA concentration $c_{D N A}$ (the DNA concentration in solution free of multivalent ions); ii) the decondensation thresh-

old $C_{d}$ of multivalent ions, when the condensed DNA returns back to solution, is almost independent of monovalent salt concentration $c_{s}$; iii) between the threshold values $C_{c}$ and $C_{d}$ the DNA fragments show two coexisting liquid crystalline phases. Concerning the first 
item, the onset of DNA condensation is usually explained by the correlation attraction between almost neutral structures. Thus, the precipitation induced by trivalent or tetravalent ions is not a consequence of the intrinsic structure and flexibility of DNA, but is a common feature of a polyelectrolyte solution. The threshold value $C_{c}$ grows with increasing monovalent salt concentration $c_{s}[2,17-19]$. A mono-molecular DNA collapse into a neutral toroidal structure occurs in highly dilute solutions of long DNA chains [3,9], whereas a multimolecular aggregation is generally observed in more concentrated DNA solutions, regardless of the DNA length $[20,21]$. The second item, the DNA redissolution at $C_{d}$, is currently under intensive debate in the colloidal community with different, and sometimes conflicting, explanations $[12,13,16,17,22]$. For instance, in Ref. [12] it is argued that after precipitation the increased binding of polyamines will make the DNA hydrophilic enough to dissolve in water. Other experiments [22] show that the DNA is still in a condensed state when polyions are added beyond the threshold concentration $C_{d}$, but it is more finely dispersed in the solution. In Ref. [17] the reentrance is explained by resorting to DNA overcharging phenomena, which take place roughly in the middle of the condensation plateau. DNA is claimed to experience negative electrophoresis and move opposite to the conventional direction near the reentrance transition. However, experiments of Raspaud et al [9] do not confirm this claim. In Ref. [13] it was suggested that the redissolution is very sensitive to the relationship between the condensation of multivalent counterions on the polyelectrolyte and multivalent counterion-monovalent coion association (Bjerrum pairs). Thus, if the chemical potential of the multivalent counterions is low, they more likely adsorb on the DNA and overcharge it. On the other hand, if the chemical potential is high, the counterion-coion associations are more likely to appear in solution. A resulting condensation of Bjerrum pairs creates consecutive layers of oppositely charged ions around the DNA molecule [14,23].

While the first two above mentioned items have been studied in considerable detail, much less attention has been paid to the third item, namely the coexistence of two different liquid-like structures in DNA condensates. In a series of experiments, Livolant and colleagues demonstrated that the spermidine and spermine ions are capable of provoking 
several liquid crystalline forms of fragmented DNA [11]. Similar results were published recently by Saminathan et al in Ref. [3].

In this paper we investigate the condensation and redissolution of DNA on a molecular level by using computer simulations of the primitive-model electrolyte with explicit tetravalent counterions and monovalent salt ions. We trace back the condensation and redissolution to the distance-dependent effective potential $U(R)$ between two parallel DNA molecules, where $R$ is the radial distance between their two centers. Using two-dimensional liquid-state theory for the fluid and lattice sums for the solid phases, we calculate the phase diagram for columnar DNA assemblies. A previous account of the results was already published elsewhere [28].

The remainder of this paper is organized as follows. In Section II we describe our model system and give the definition of DNA-DNA interaction forces. We calculate the interaction forces for different counterion and salt concentrations in Section III. Section IV is devoted to the free energy perturbation theory for defining the liquid-liquid coexistence densities. We conclude in Section V.

\section{THE MODEL}

We consider a B-DNA molecule which is a double helix with a pitch length $P=34 \AA$. There are $N_{p}=20$ phosphate charges per one helical turn which makes one elementary charge per each $1.7 \AA$ of axial rise. The geometrical shape and charge distribution of DNA is modeled through the accurate Montoro-Abascal model (MAM) [24,25]. Its cross section is illustrated in Fig. 1.

A pair of DNA molecules are placed along the $x y$ diagonal of the cubic simulation box of volume $V=L^{3}$. The size of the simulation box $L=102 \AA$ corresponds to three full turns of DNA. The box also consists of $N_{Q}$ multivalent ions with valency $q_{Q}, N_{-}=N_{s-}+q_{Q} N_{Q}$ monovalent coions and $N_{+}=N_{s+}+N_{c}$ monovalent counterions. Here $N_{s+}=N_{s-}=N_{s}$ is

the number of added salt ion pairs, $N_{c}$ is fixed by the DNA phosphate charge due to the 
constraint of global charge neutrality $\left(N_{c}=120\right.$ in our simulations). All ions are modelled as charged hard spheres. Tetravalent counterions are assumed to represent spermine ions. Though the latter is a polyion in vivo, a spherical approximation can be used, since the bibliographical data support the idea that it is the charge of counterion, rather than its structural specificities, which is important in DNA condensation and redissolution processes [9]. Beside of this, the fluidity of the ordered DNA phase also suggests that spermine binds like an ordinary ion to the DNA surface, rather than forming inter-strand cross-links to neighboring DNA molecules [11,12]. Assuming that the ions of the same valency are indistinguishable, the actual number of mobile ion species in the simulation box is reduced from five (which are multivalent ions and their coions, positive and negative monovalent salt ions and monovalent counterions that neutralize the DNA phosphate charge) to three: multivalent counterions (charge $q_{Q}=4$ and diameter $d_{Q}$ ) and positive and negative small ions (charge $q_{c}= \pm 1$ and diameter $d_{c}$ ). Periodic boundary conditions in all three directions are applied to reduce finite size effects. The DNA replicas in the $z$ direction produce infinitely long molecules. The whole system is held at room temperature $T=298 K$ and the water is modelled as a continuous dielectric medium with dielectric constant $\epsilon=80$. A typical snapshot of the simulation is shown in Figure 2.

The interaction potentials between the five sort of particles (three of them are the mobile ions in solution mentioned above, and two of them, one charged and the other neutral, belong to the DNA molecule, see Figure 1) are a combination of hard core and Coulomb potentials:

$$
V_{i j}(r)= \begin{cases}\infty & \text { for } r \leq\left(d_{i}+d_{j}\right) / 2 \\ \frac{q_{i} q_{j} e^{2}}{\epsilon r} & \text { for } r>\left(d_{i}+d_{j}\right) / 2,\end{cases}
$$

Here $r$ is the inter-particle distance, $i, j=Q$ for multivalent counterions, $c$ for small ions, $p$ for phosphate charges and $n$ for neutral spheres in the MAM (with $q_{n}=0$ ). There is also an excluded volume potential $V_{i}^{0}$ between the DNA hard cylinder and the free ions $i, j=Q, c$.

The basic quantity of interest is the effective force per helical turn [26]

$$
\vec{F}=\vec{F}_{1}+\vec{F}_{2}+\vec{F}_{3}
$$


between two DNA molecules. Here $\vec{F}_{1}$ is the direct Coulomb repulsion per helical turn of one DNA molecule as exerted from the phosphate groups of the other DNA. It does not depend on salt density and its calculation is straightforward [27]. The second term $\vec{F}_{2}$ in Eq. (2) corresponds to Coulomb interactions between the phosphate charges at positions $\vec{r}_{k}^{p}$ $\left(k=1, . . N_{p}\right)$ and the mobile ions at positions $\vec{r}_{l}^{i}\left(l=1, . . N_{i}, i=c, Q\right)$,

$$
\vec{F}_{2}=-\frac{1}{3} \sum_{k=1}^{3 N_{p}}\left(\left\langle\sum_{i=c, Q} \sum_{l=1}^{N_{i}} \vec{\nabla}_{\vec{r}_{k}{ }^{p}} V_{p i}\left(\left|\vec{r}_{k}^{p}-\vec{r}_{l}^{i}\right|\right)\right\rangle\right) \text {. }
$$

Here $<\ldots>$ denotes canonical average over small ion configurations. The third term $\vec{F}_{3}$ in Eq. (2) arises from the entropic contribution of small ions due to their moment transform to the DNA surface $\mathcal{S}$,

$$
\vec{F}_{3}=-\frac{1}{3} k_{B} T \int_{\mathcal{S}} d \vec{f}\left(\sum_{j=c, Q}\left\langle\rho_{j}(\vec{r})\right\rangle\right) .
$$

Here $\vec{f}$ is a surface normal vector pointing outwards the DNA core and $\rho_{j}(j=c, Q)$ is the inhomogeneous microion concentration. The canonical averages in $\vec{F}_{2}$ and $\vec{F}_{3}$ are carried over all configurations of the mobile ions.

We have performed extensive grand-canonical molecular dynamics (GCMD) simulations, similar to those of Ref. [27], for a range of different tetravalent counterion and monovalent salt concentrations. Each simulation is referred by its nominal tetravalent counterion concentration $C$ (salt ion concentration $c_{s}$ ) defined as a ratio between the total number of ions $N_{Q}\left(N_{s}\right)$ in the cell without the DNA molecules and the system volume $V, C=N_{Q} / V$ $\left(c_{s}=N_{s} / V\right)$. Additional simulations have been carried for these bulk phases in order to calculate the chemical potential $\mu$ of solution. Then in the simulations with DNA molecules the number of ions in the simulation cell was automatically adjusted to the specified value of chemical potential $\mu$. The ion diameter was chosen to be $d_{Q}=8 \AA$ for tetravalent counterions and $d_{c}=4 \AA$ for other free ions in the system. This parameter defines the closest approach of the ion to the DNA surface and has a strong impact on the polyion electrostatics. The time step $\triangle t$ of the simulation corresponds to an average ion displacement of $0.03 \AA$ per time step such that the reflection of counterions following the collision with the combined surface of 
DNA is calculated with high precision. About $5 \times 10^{4} \mathrm{MD}$ steps are required on average to reach equilibrium. The time evolution is then followed for $5 \times 10^{4}-5 \times 10^{6}$ steps to gather statistics to calculate canonical averages of the interaction forces.

\section{RESULTS FOR THE INTERACTION FORCES}

The case of a single DNA molecule in the presence of spermine ions has been considered in Ref. [28]. It was shown there (see Figure 2 in Ref. [28]) that the ionic cloud may not only compensate the polyion charge but even exceed it, resulting in an opposite sign of the electrostatic potential at some distances from the DNA surface. The adsorption of Bjerrum pairs [23] onto the DNA surface at high tetravalent counterion concentration creates consecutive layers of charges of different sign around the DNA molecule. The onset of a multilayer structure occurs at $C=1.8 \mathrm{mM}$. Addition of monovalent salt shifts this threshold concentration to lower values of $C$, in accordance with experimental observations and twocomponent Manning condensation theory [29]. For multivalent counterion concentrations exceeding $C=1.8 \mathrm{mM}$, the total charge in the DNA grooves remains constant and only the total ionic charge adsorbed on the strands contributes to the overcharging, similar to our earlier findings $[25,28]$. Beside of this, there is a competition between the multivalent and monovalent counterions in binding to the DNA surface as $C$ increases. The multivalent ions tend to replace the monovalent counterions on the DNA surface. Thus, at higher $C$ the main contribution to the formation of charged layers around DNA molecules comes from Bjerrum association between big counterions and small coions. Such charged layers give rise to an attraction between two parallel DNA molecules, as shown in Figure 3. A decrease of the big counterion charge leads to the break-up of the Bjerrum counterion-coion pairs and thus destroys the layer formation around the DNA molecule. This ultimately results in the loss of intermolecular attraction, see simulation data for $q_{Q}=1,2$ and 3 in Figure 3.

It should be mentioned that in addition to the intermolecular (or axis-to-axis) distance $R$, there are angular variables which define the mutual configuration of two parallel DNA 
molecules corresponding to the orientation of their grooves and strands [26]. At short surfaceto-surface distances between the two DNA molecules up to $5 \AA$ there are strong contributions from particular DNA-DNA configurations [27]. For larger separation distance, $R>25 \AA$ we find no detectable dependence of the interaction forces on the mutual orientations of DNA molecules. On the other hand, there is experimental evidence that in DNA condensates two neighboring molecules never approach each other closer than $5 \AA$. This apparently means that at such small distances a strong repulsion between DNA solvation shells exists. Thus in all figures hereafter we show orientationally averaged interaction forces starting from the distance $R=24 \AA$. The interaction forces are scaled per DNA pitch, i.e. per 10 DNA base pairs.

The electrostatic $F_{2}$ and entropic $F_{3}$ components of total interaction force $F$ for tetravalent counterions corresponding to DNA overcharging are separately plotted in Figure 4. It can be seen that the electrostatic force shows oscillations around zero, which are reminiscent of the multilayered structure of charges around a single DNA molecule [28]. The deep attractive minimum of the total force $F$ has an entropic origin, whereas the second minimum at intermolecular distance $\mathrm{R}=41 \AA$ has a purely electrostatic origin.

In Figure 5 we plot the DNA-DNA interaction force for both the undercharged and overcharged cases at different salt concentrations. It is evident that in dense salt solutions the attractive minimum becomes weaker. For overcharged DNA, $C=65 \mathrm{mM}$, the position of the second minimum shifts towards the DNA surface. Thus, whereas the DNA overcharging does not strongly depend on the added salt concentration $c_{s}$ [28], the effective forces do.

The positions of the minimum and the maximum of the force shift towards the DNA surface also for higher spermine concentrations. This trend is shown in Figure 6, where the DNA-DNA interaction force is plotted for different spermine and salt concentrations at fixed distance $R=38 \AA$. For low $C$, which in Figure 6 corresponds to the area to the left of point $\mathrm{A}$, the DNA-DNA interaction is overall repulsive. Between the points $A$ and $B$ a first minimum develops in $F$. As the spermine concentration increases further, the minimum shifts toward the DNA surface and the force has a repulsive tail. This tail indicates the 
appearance of second maximum, which obviously is followed by second minimum.

The dependence of the DNA-DNA interaction force on $C$ for two fixed intermolecular distances are shown in Figure 7. Five different arrows in this picture point to different values of $C$ which characterize the number of attractive minima in the interaction force $F$. For small $C=0.01 \mathrm{mM}$, indicated by the arrow next to $a$ in Figure 7 , the interaction force has no minimum and thus is totally repulsive. For spermine concentration $C=0.025 \mathrm{mM}$, corresponding to the arrow next to $b$, the force has a single minimum. For intermediate $C=1.7 \mathrm{mM}$ and $C=56 \mathrm{mM}$, see the arrows next to $c$ and $d$ respectively, there are two minima in the interaction force (note that a positive force at $R=38 \AA$ for $C$ corresponding to arrow $d$ indicates the occurrence of second maximum, which obviously if followed by second minimum at larger $R$ ). And finally, at even higher $C=400 \mathrm{mM}$, the first minimum has disappeared, however the second minimum is retained.

A full set of distance resolved DNA-DNA interaction force curves for different tetravalent counterion densities $C$ are presented in Figure 8. It can be seen that even a small trace of spermine ions - well below the overcharging threshold - induces an attraction between the DNA molecules, except at very close distances, see curve for $C=0.1 \mathrm{mM}$. As the DNA molecules get more overcharged, which corresponds to high spermine concentrations, the main minimum narrows and becomes more shallow. At the same time, the width and the height of the maximum increases. We note that the attractive minimum for undercharged DNA pair has mainly a pure electrostatic origin and arises due to charge correlations in the electrolyte. However, for overcharged DNA the main contribution to the force at this minimum is due to the spermine crowding near the DNA surface. The second maximum originates both from electrostatic and entropic forces. Finally, the second minimum emerges from a pure electrostatic effect.

In the following we calculate the total effective pair interaction potential $U(R)$ per unit length for a given bulk salt concentration $c_{s}$ and different Spe concentrations $C$. The quantity $U(R)$ is obtained by integrating the distance-resolved interaction force averaged over all microion configurations [34]. Results are shown in Figure 9. The oscillations in the force 
imply that the interaction potential also oscillates. With increasing $C$, the first minimum of $U(R)$ is getting deeper and is achieving a maximal depth at the overcharging concentration $C \approx 1.8 \mathrm{mM}$. A further increase of $C$ again reduces the depth of this minimum. The position of the minimum, on the other hand, hardly depends on $C$. The Spe-layering around the pair of DNA molecules induces a second minimum at larger separations as revealed in the enlarging inset of Figure 9. This minimum is of electrostatic origin and occurs for $C \gtrsim 65 \mathrm{mM}$. Again the depth of the second minimum first increases and then decreases with increasing $C$. At intermediate Spe concentrations, we are thus confronted with a double minimum potential which is induced by layering. It is worth to mention that positions of the second minimum can be related to intermolecular distances between the DNA molecules in cholesteric phase induced by polyamines [32,3]. Direct measurements [30] and theoretical investigations [31] of intermolecular forces demonstrated that the energetics of this cholesteric phase is determined primarily by electrostatic interactions. Since the potentials in Figure 9 are scaled for one DNA pitch length, the interaction strength corresponding to the minimum of curve (2) for very long DNA molecules is sufficiently large to induce condensation. This implies that DNA aggregation can take place well below overcharging Spe concentrations.

\section{PHASE DIAGRAM FOR DOUBLE-MINIMUM POTENTIAL.}

The characteristic double-minimum structure of the interaction potential $U(R)$ will give rise to an unusual phase behavior. We have calculated the phase diagram of a columnar DNA assembly on the basis of our simulated effective pair interactions. To do so, we assume that the DNA molecules are parallel along a certain length $\ell$. This length is an additional parameter which we fix to be $\ell=20 \times P$. We comment on the dependence of the phase diagram on $\ell$ in Section VI. The assembly of parallel DNA can be considered as a twodimensional many-body system interacting via a potential $\ell \times U(R)$ and being characterized by a DNA particle number density $\rho$. We calculated the free energies of the fluid and solid phases by using different techniques outlined below and perform the traditional Maxwell 
double tangent construction to identify the coexisting densities.

The free energy of dilute fluid phase is approximated by the two-dimensional perturbation theory [35] via splitting the total potential into a repulsive and attractive parts, $U(R)=$ $U_{r}(R)+U_{a}(R)$. The repulsive potential $U_{r}(R)$, identical to $U(R)$ but truncated and shifted towards zero at the first minimum at $R=R_{\text {min }}$, is mapped onto that of effective hard disks of diameter $\sigma_{\text {eff }}[36]$

$$
\sigma_{e f f}=\sigma+\int_{\sigma}^{R_{\min }}\left[1-\exp \left(-\frac{U_{r}(R)}{k_{B} T}\right)\right] d R .
$$

Here the cross-section diameter for the DNA molecule is $\sigma=20 \AA$. The total Helmholtz free energy involves that of a hard disk fluid with effective area fraction $\eta=\frac{\pi \rho \sigma_{e f f}^{2}}{4}$ and a mean-field correction which we simply model as $\pi \rho^{2} \int_{\sigma}^{\infty} \frac{U_{a}(R)}{k_{B} T} R d R$. For the free energy of a hard-disc fluid, analytical expressions are available [37]. The free energy of the solid phase, on the other hand, is calculated as a lattice sum with the assumption of a two-dimensional triangular lattice structure. The lattice constant is directly related to the DNA number density $\rho$.

Figure 10 shows the resulting phase diagram with the coexisting DNA densities for the wide range of $C$. At low $C$ there is a strong first-order gas-crystal phase transition whichs coexistence region is widened for larger $C$ due to the increasing attractions. Between the two threshold concentrations $C \approx 0.1 \mathrm{mM}$ and $C \approx 65 \mathrm{mM}$ there is enough attraction to stabilize a liquid phase of high DNA density. At higher Spe concentrations a second crystal, with a considerable larger lattice constant than that of the high-density solid emerges. We call this novel phase a mesocrystal since its density is intermediate between that of the fluid and the almost closed-packed solid.

Condensation (see the cross in Figure 10) and subsequent redissolution (dot-dashed line in Figure 10) of dilute DNA solution are other implications of phase diagram. As the spermine concentration increases for fixed $\rho \sigma^{2}=0.002$, which corresponds to a typical DNA concentration of $1 \mathrm{mg} / \mathrm{ml} \mathrm{DNA}$, first the gas-liquid coexistence line is encountered. This is the condensation transition and the system will split into a low density gas and a high 
density liquid phase. At much higher $C$ the coexistence line is touched again and the system redissolves back into the dilute gas phase. The corresponding threshold concentrations of the condensation and redissolution are in the range $C_{c} \approx 0.3 \mathrm{mM}$ and $C_{d} \approx 165 \mathrm{mM}$ and agree well with the experimental observations $[9,11]$.

\section{DISCUSSIONS AND CONCLUSIONS}

One issue we want to discuss is the dependence of the phase diagram on the DNA length $\ell$. Since $\ell$ is a prefactor of the effective potential, it plays formally the role of an inverse system temperature. We have explored the phase behavior for smaller and larger DNA segment lengths of $\ell=5 P$ and $\ell=100 P$ respectively. Firstly, the stability of liquid pocket depends sensitively on $\ell$; it disappears completely for small $\ell$, but extends towards larger $C$ for larger $\ell$. Secondly, the fluid coexistence density shifts to considerably higher values for smaller $\ell$. Hence, condensation and redissolution is prohibited for small DNA-segment lengths. This is in line with the experiments of Ref. [38], where a threshold value of $\ell \approx 15 P$ for the minimal length $\ell$ required for condensation is reported. For a triplex DNA (three stranded DNA molecule) the minimal length is reported to be about $2 P$ [38]. The difference between the minimal lengths for duplex and triplex DNA segments arises from the DNA

charge density [39,40]. The higher the linear charge density, the stronger the overcharging. This in turn results in the precipitation of triplex DNA at spermine concentrations, where duplex DNA does not aggregate [38,12].

We speculate that the height of the energetical barrier between the two minima in the interaction potential $U(R)$ could regulate the DNA segment lengths in the crystalline structures observed in experiments. Imagine that the solution consists of a mixture of DNA segments of different lengths (but still smaller than the persistence length $500 \AA$ ). At lower spermine concentration, when the interaction potential has a single minimum, all DNA segments will form a bundle with an average inter-particle distance of the order of $R=28 \AA$. However, when the energetical barrier develops at intermediate spermine concentrations 
with a height around $0.5 k_{B} T-1 k_{B} T$ per pinch length, only short DNA segment could overcome this repulsion. Thus, short segments will fall into first minimum and form a dense hexagonal structure, while longer DNA segments will be trapped in the second minimum and form more swollen, fluid-like structure, apparently a cholesteric phase.

The other issue is the orientational entropy of DNA molecules and its influence on the interaction potential and free energy of DNA solutions. For higher DNA concentrations, due to the strong attraction (more than $40 k_{B} T$ ) between the DNA rods of 20P length, all other than parallel configurations of DNA molecules have little statistical weight and can therefore be neglected in the configurational averaging of the interaction potential. For the dilute gas phase the orientational entropy for DNA rod has an upper limit of about $2 k_{B} T$. A corresponding upward shift of the free energy of dilute DNA system will not strongly affect the coexistence spermine concentration values deduced from the double-tangent procedure.

In conclusion, we have calculated the influence of tetravalent counterions on the effective interactions and the phase diagram of columnar DNA assemblies by primitive-model-type computer simulations and statistical theories. We find that a small concentration of tetravalent counterions induces DNA condensation. The layering of the strongly coupled tetravalent counterions on the DNA strands yields an oscillatory effective interaction potential with a double-minimum structure at intermediate counterion concentrations. This explains the redissolution transition and triggers a novel stable mesosolid. Our threshold concentrations are in good agreement with experimental data.

\section{ACKNOWLEDGMENTS}

We acknowledge a partial support of this work by the European Networks of Excellence 'SoftComp'. 


\section{FIGURES}

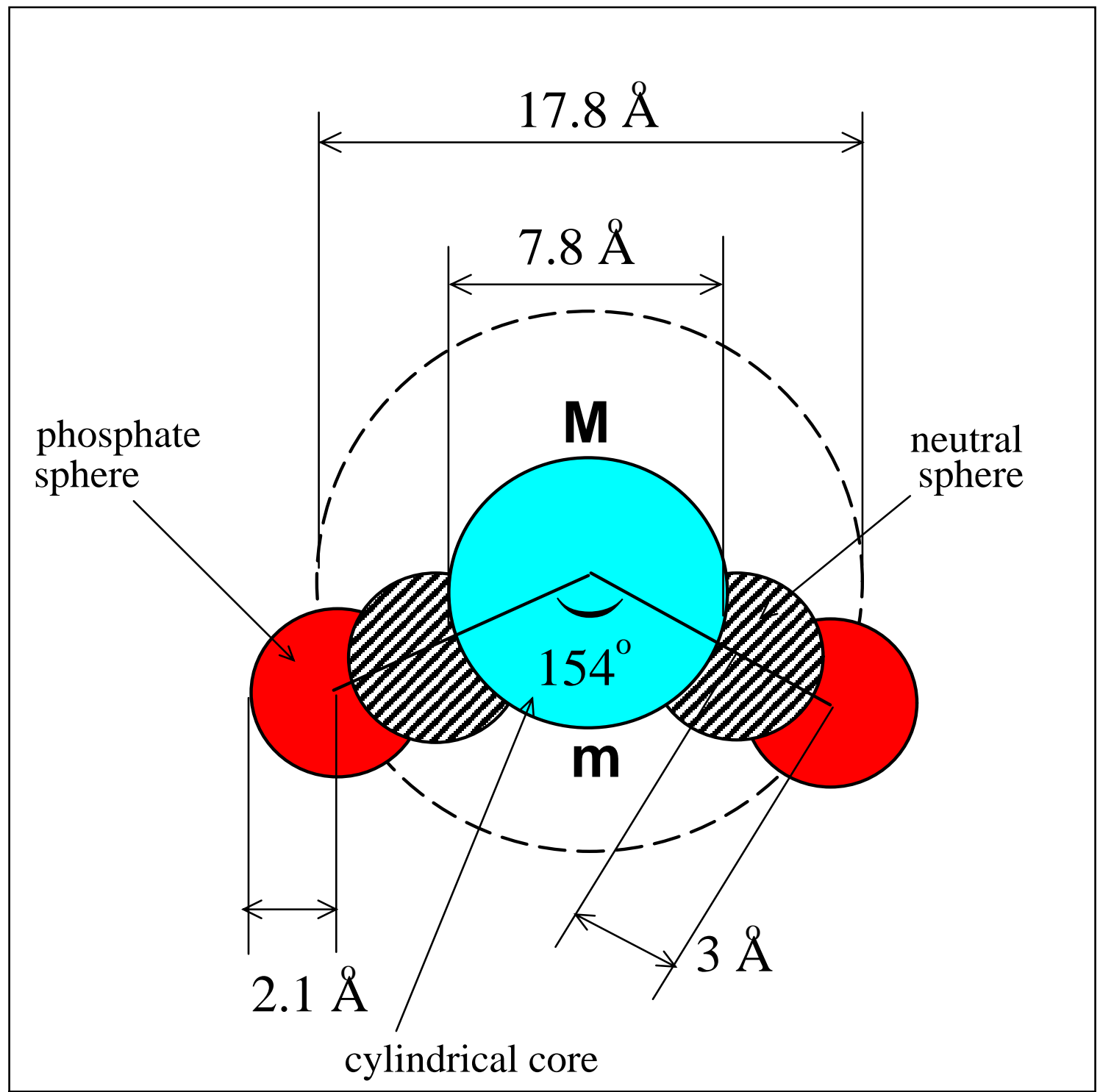

FIG. 1. (Color online) Cross section of DNA in the $x y$ plane for the Montoro-Abascal model (MAM). Phosphate charges are shown as dark spheres. The DNA cylindrical core is colored in gray, the hatched areas correspond to neutral hard spheres. The inscribed letters "M" and "m" denote the major and minor grooves correspondingly. 


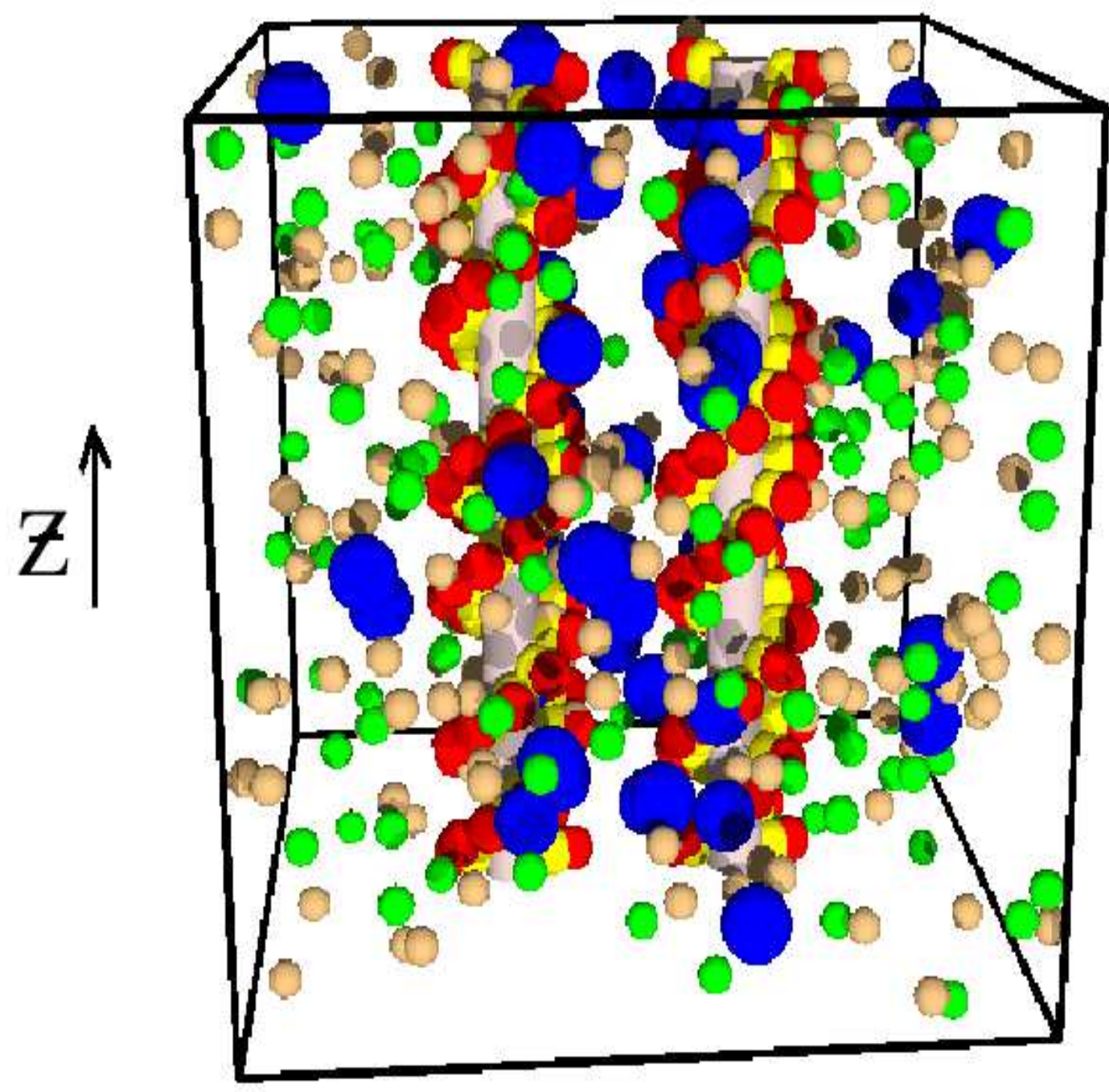

FIG. 2. (Color online) Typical snapshot in the simulation cell. The DNA molecules are shown as two parallel rods in the $z$ direction, over-wrapped by two strings of light grey (neutral sphere in MAM (see text), colored yellow in online figure) and grey (phosphate sphere in MAM, red in online figure) spheres. The tetravalent Spe ions are shown as big black (blue in online figure) spheres. Light grey (yellow in online figure) spheres represent coions, and dark grey (green in online figure) spheres are monovalent counterions. 


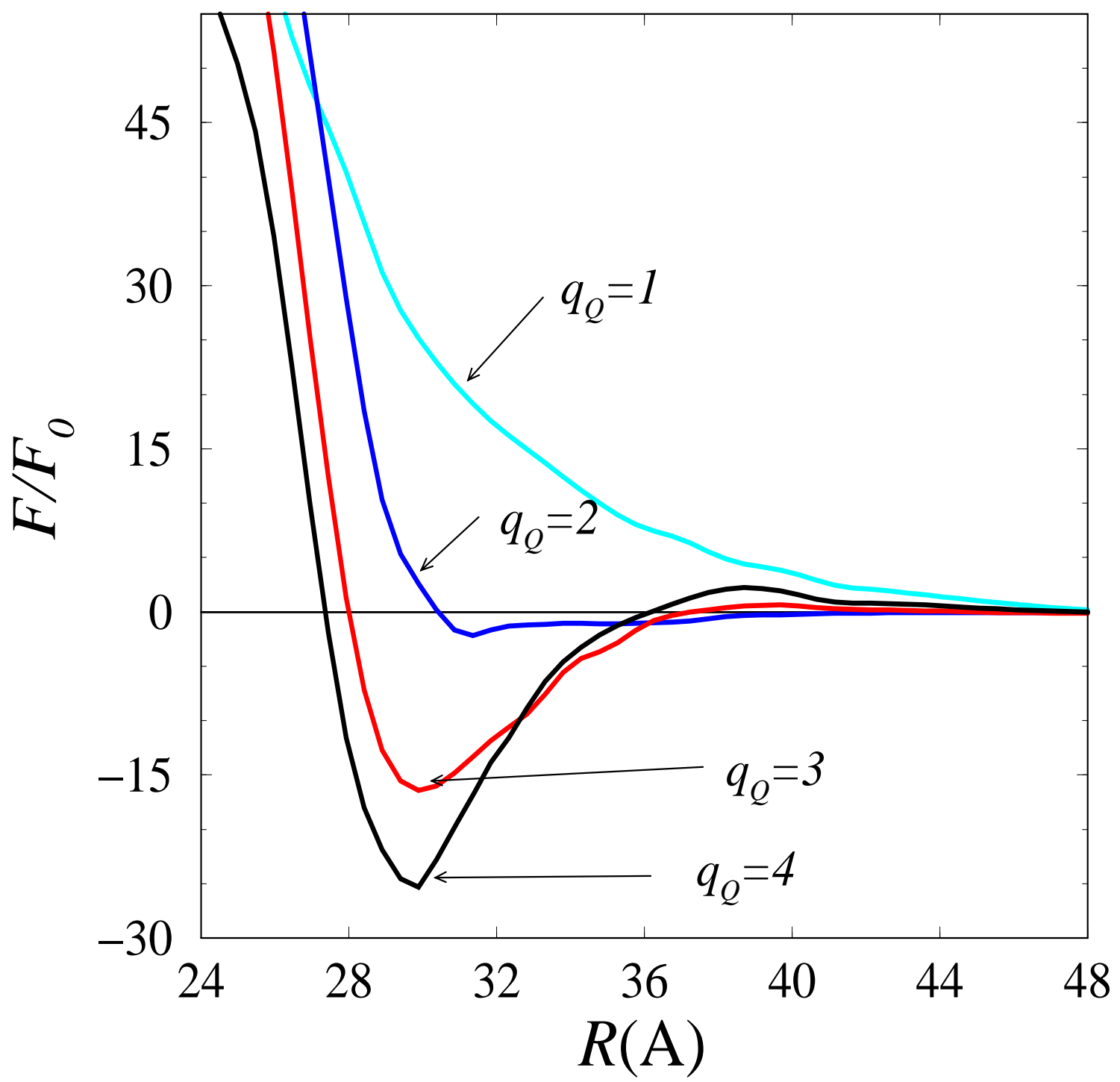

FIG. 3. (Color online) DNA-DNA interaction force $F / F_{0}$ versus intermolecular separation distance $R$ for $c_{s}=25 \mathrm{mM}$ and $C=65 \mathrm{mM}$. The charge of the big counterions is indicated next to the corresponding curves. $F_{0}=k_{B} T / P$, where $P=34 \AA$. 


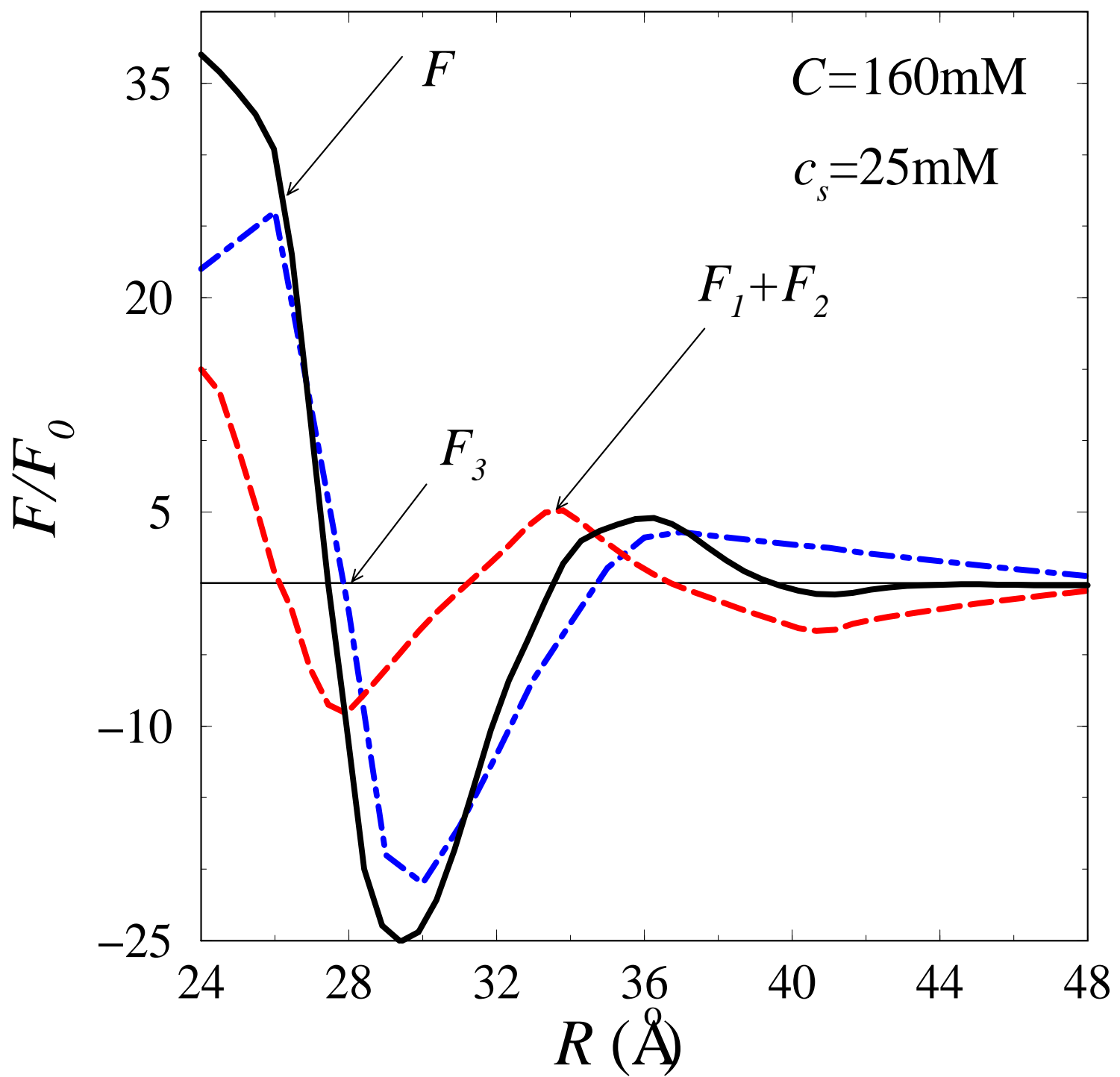

FIG. 4. (Color online) DNA-DNA interaction force $F / F_{0}$ versus intermolecular separation distance $R$ for $c_{s}=25 \mathrm{mM}, C=160 \mathrm{mM}$. $F_{0}=k_{B} T / P$, where $P=34 \AA$. The parameters corresponds to complete DNA overcharging. Note that there is a second maximum in the total force $F$ at about $R=36 \AA$ and a second minimum at about $R=41 \AA$. 


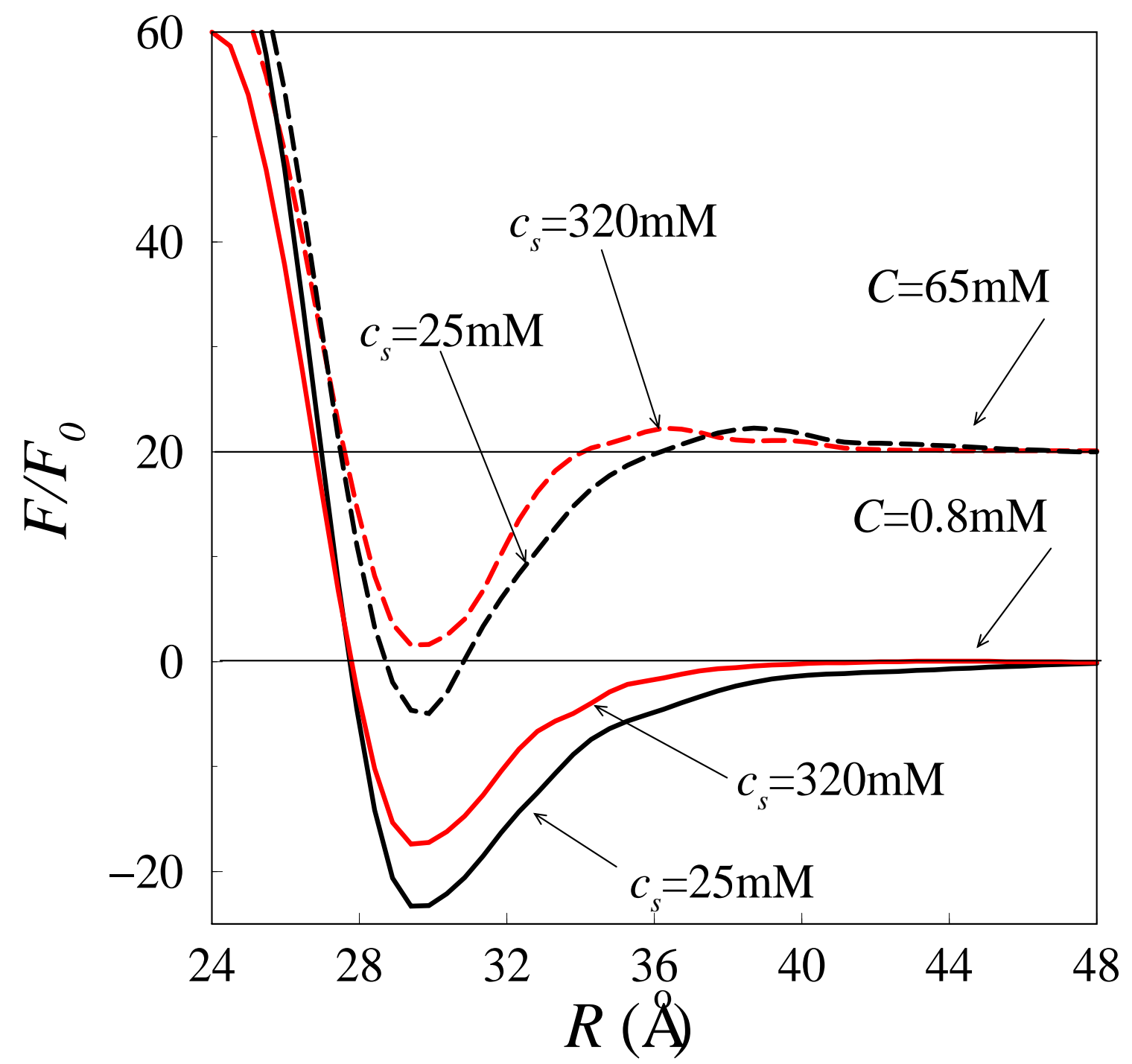

FIG. 5. (Color online) DNA-DNA interaction force versus intermolecular separation distance $R$ for $c_{s}=25 \mathrm{mM}$ (black lines in online figure) and $c_{s}=320 \mathrm{mM}$ (red lines in online figure) and two different spermine concentrations: $C=0.8 \mathrm{mM}$ (undercharged DNA, solid lines) and $C=65 \mathrm{mM}$ (overcharged DNA, dashed lines). $F_{0}=k_{B} T / P$, where $P=34 \AA$. The curves that correspond to $C=65 \mathrm{mM}$ are shifter upward for clarity. 


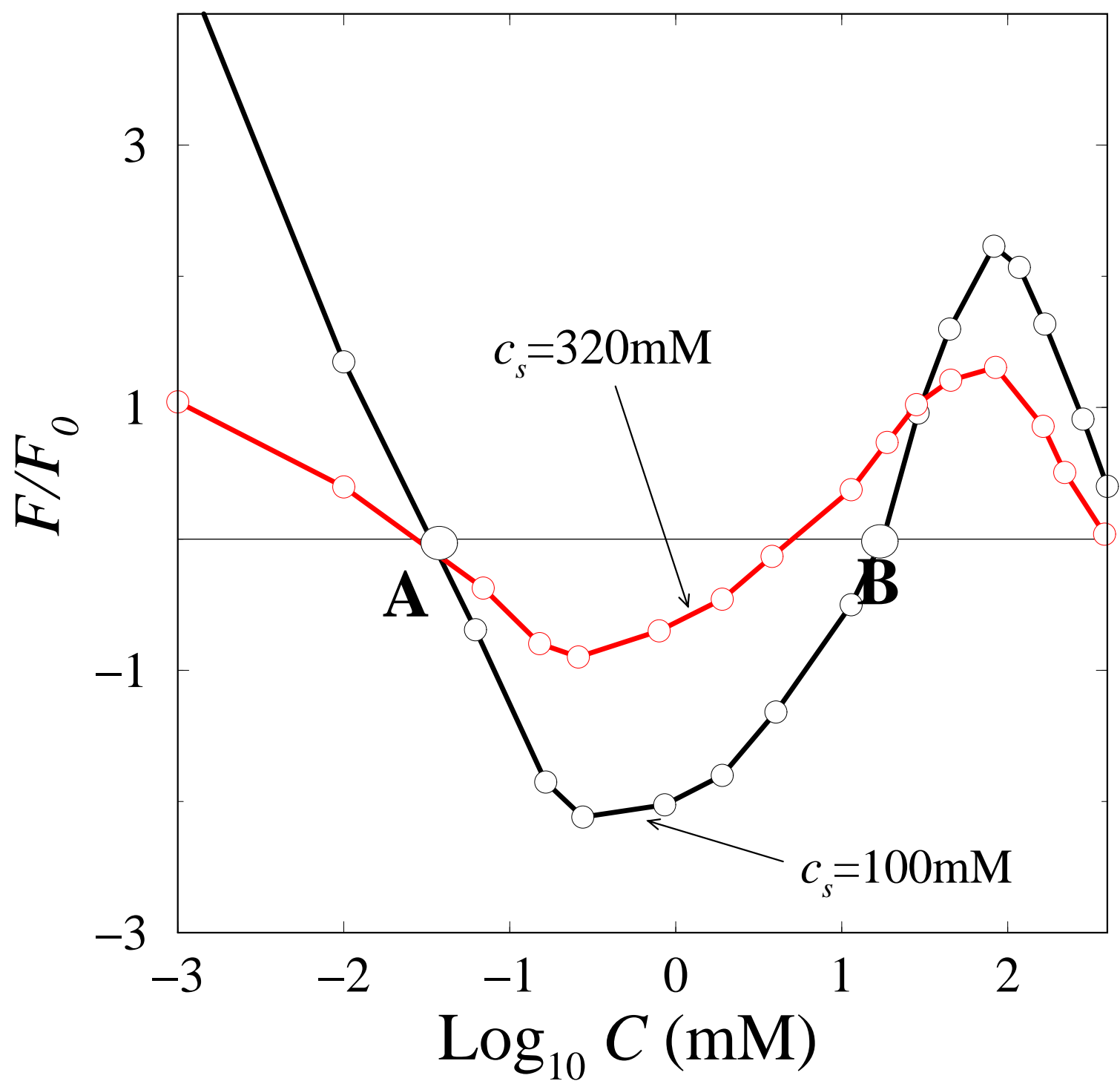

FIG. 6. (Color online) DNA-DNA interaction force versus spermine concentration $C$. The intermolecular separation distance is $R=38 \AA$ (roughly the position of the second maximum for $C=65 \mathrm{mM}$ ). In the region between the points A and B (for salt concentration $c_{s}=100 \mathrm{mM}$ ) first minimum of the interaction force develops. $F_{0}=k_{B} T / P$, where $P=34 \AA$. 


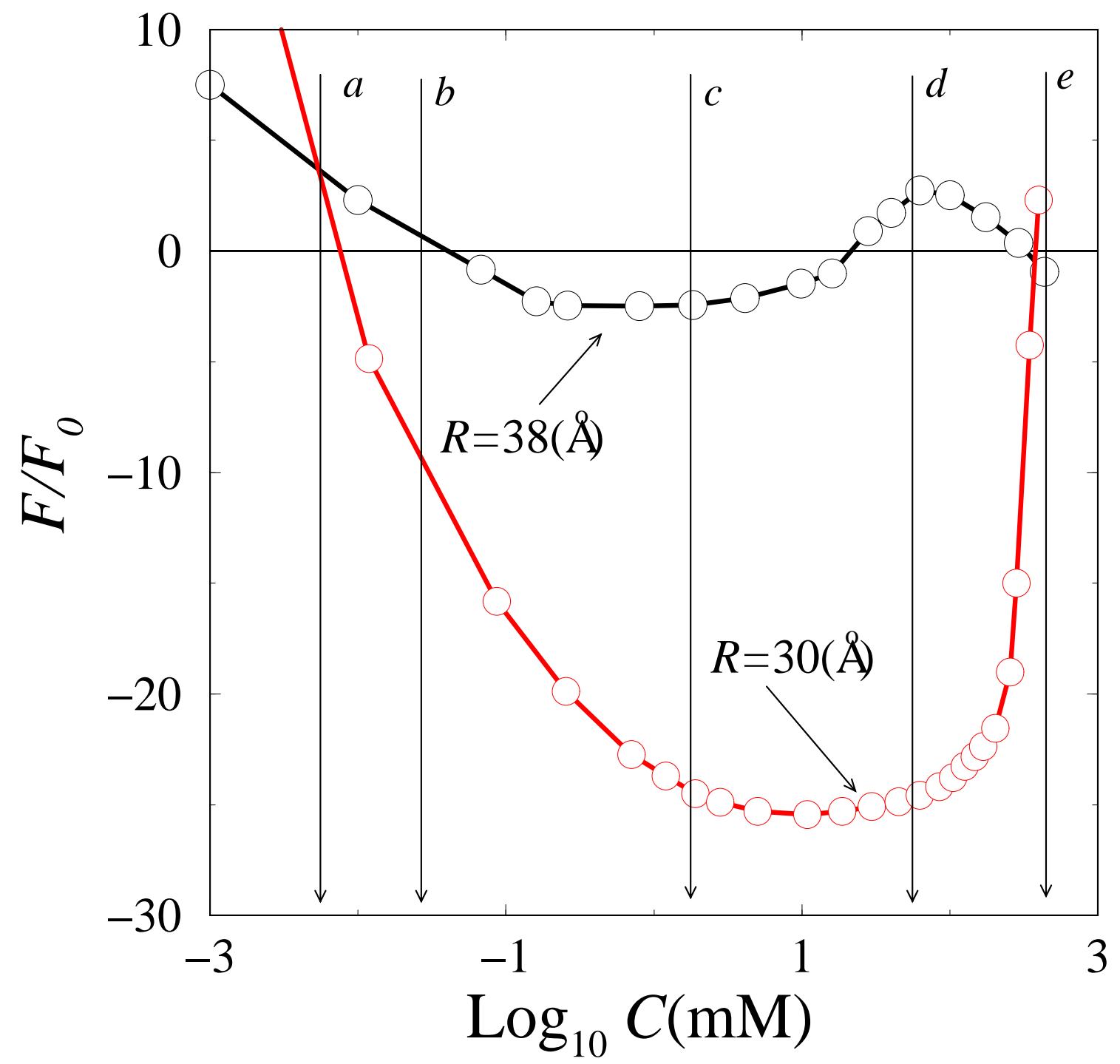

FIG. 7. (Color online) DNA-DNA interaction force versus spermine concentration $C$ at fixed intermolecular separation distances $R$ for $c_{s}=25 \mathrm{mM}$. At $R=30 \AA \quad(R=38 \AA$ ) the first (second) minimum emerges at intermediate spermine concentrations $C$ and then disappears at higher $C$. Arrows and labels from $a$ to $e$ next to them are a guide to eyes to point the different spermine concentrations where the shape of the interaction force undergoes significant changes. For more details see the text. 


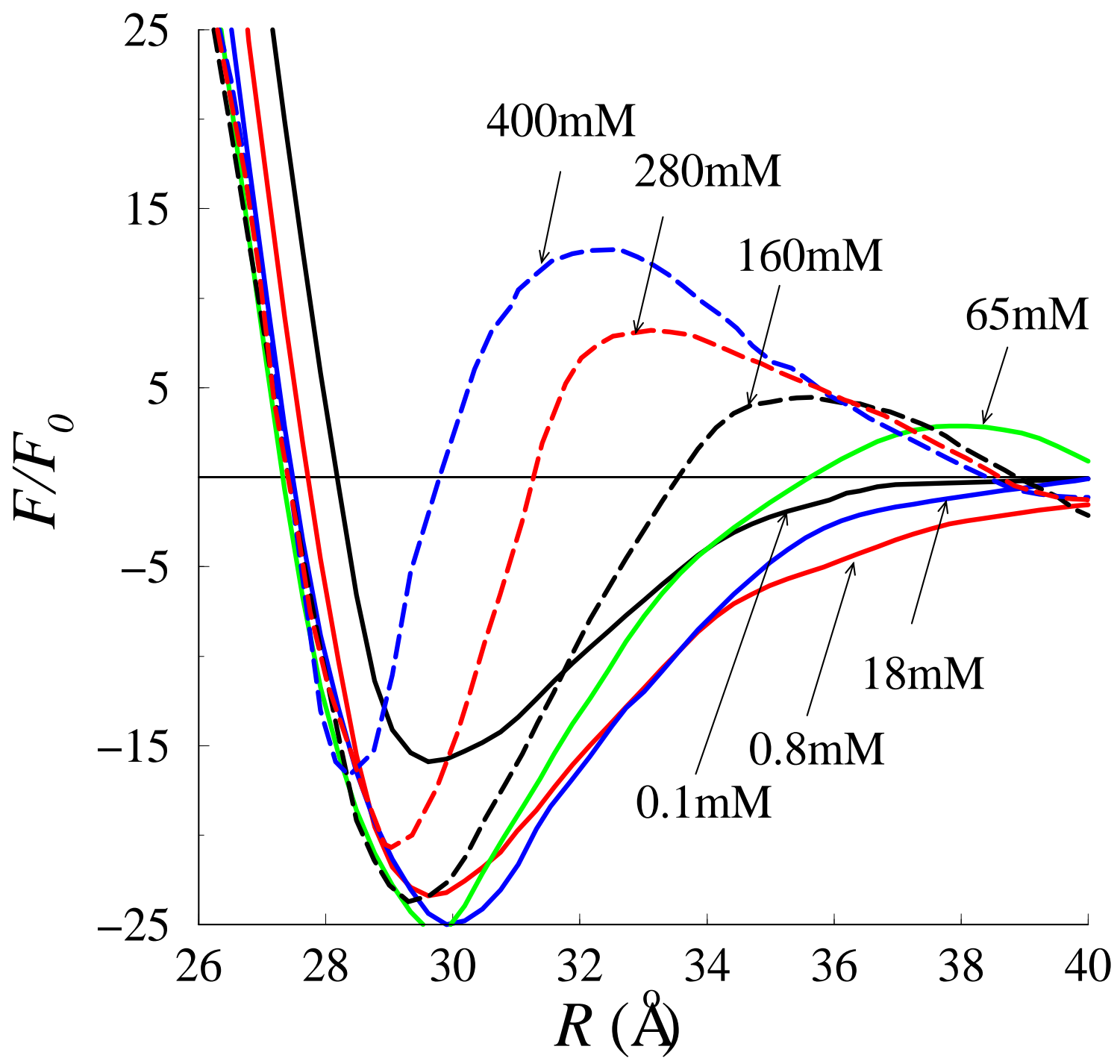

FIG. 8. (Color online) DNA-DNA interaction force versus intermolecular separation distance $R$ for $c_{s}=25 \mathrm{mM}$, Different spermine concentrations are indicated next to corresponding curves: $C=0.1 \mathrm{mM}, 0.8 \mathrm{mM}, 18 \mathrm{mM}, 65 \mathrm{mM}, 160 \mathrm{mM}, 280 \mathrm{mM}, 400 \mathrm{mM} . F_{0}=k_{B} T / P$, where $P=34 \AA$ is the DNA pitch length. 


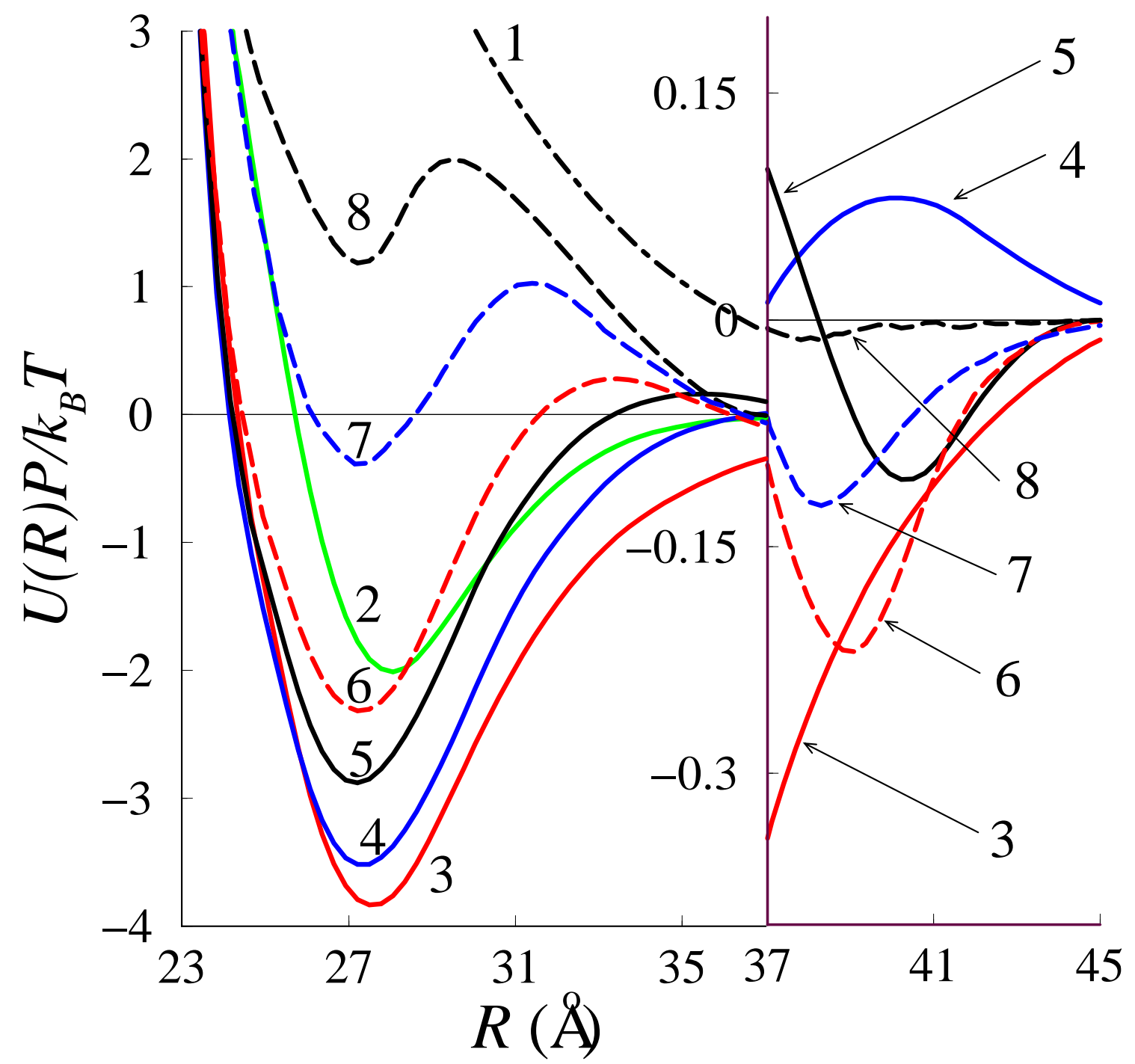

FIG. 9. (Color online) DNA-DNA effective pair potential versus intermolecular separation distance $R$ for $c_{s}=25 \mathrm{mM}$. The spermine concentrations are $C=0 \mathrm{mM}(1), 0.1 \mathrm{mM}(2), 0.8 \mathrm{mM}(3)$, $18 \mathrm{mM}(4), 65 \mathrm{mM}(5), 160 \mathrm{mM}(6), 280 \mathrm{mM}(7), 400 \mathrm{mM}(8)$. 


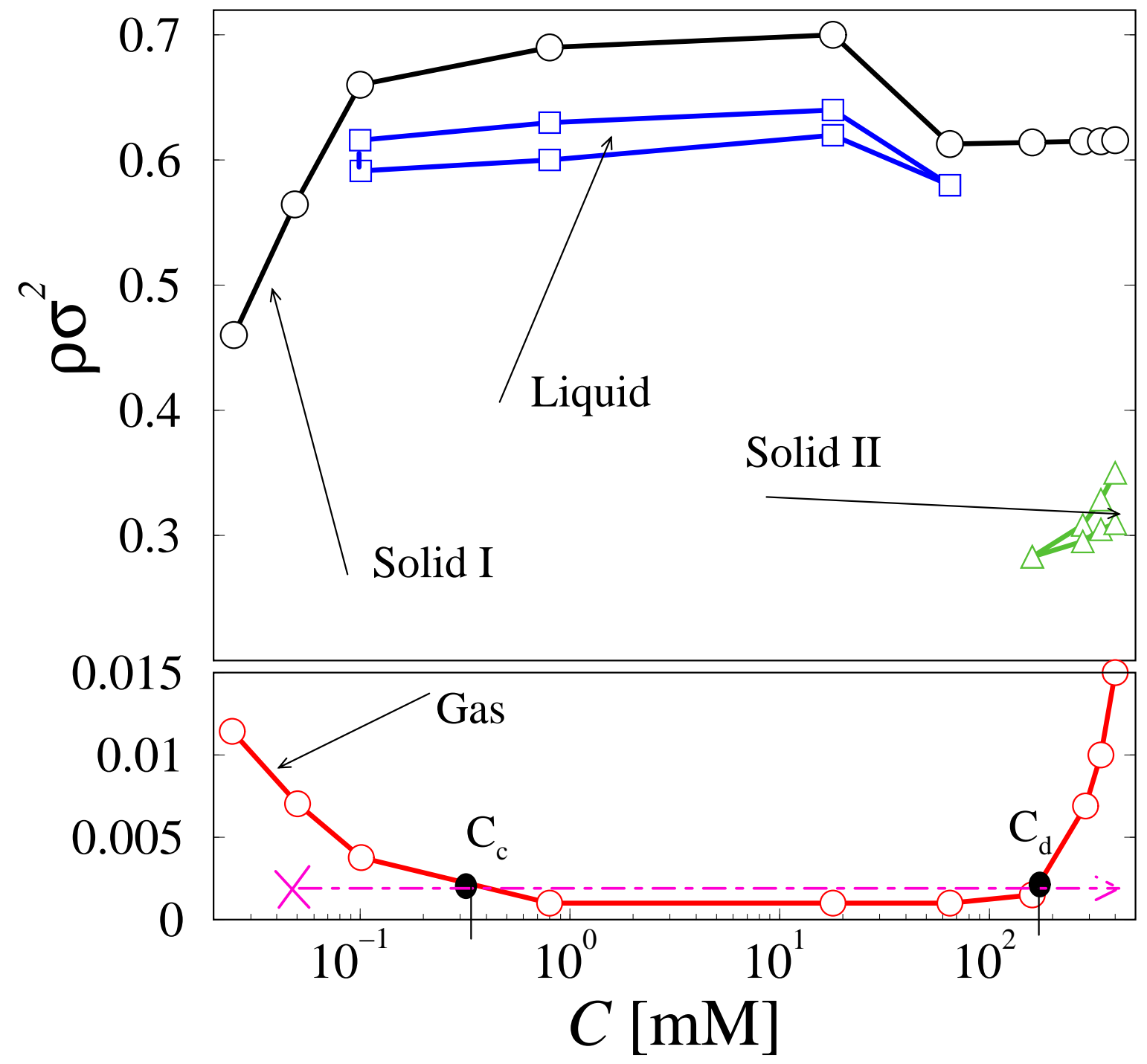

FIG. 10. (Color online) Coexisting DNA densities as a function of Spe concentrations $C$, for $c_{s}=25 \mathrm{mM}$. The stable phases found are gas-like, liquid and two triangular crystals with different lattices constants (solid I and solid II). All phase transition between these phases are of first order. For the sake of better resolution at smaller DNA densities, the $y$-axis is expanded below $\rho \sigma^{2}<0.015$ 


\section{REFERENCES}

[1] S. S. Cohen, A Guide to Polyamines, Oxford University Press, New-York, NY, 1998.

[2] V. A. Bloomfield, Biopolymers 44, 269 (1997).

[3] M. Saminathan, T. Thomas, A. Shirahata, C. K. S. Pillai, T. J. Thomas, Nucleic Acids Research 30, 3722 (2002).

[4] A.L. Cason, et al, European J. of Human Genetics 1 , 8 (2003).

[5] R. W. Wilson, V. A. Bloomfield, Biochemistry 18, 2192 (1979); R. W. Wilson, D. C. Rau, V. A. Bloomfiled, Biophys. J. 30, 317 (1980).

[6] H. Deng, V. A. Bloomfeld, J. M. Benevides, G. J. Thomas Jr, Nucleic Acids Research 28, 3379 (2000).

[7] W. H. Braunlin, C. F. Anderson, M. T. Record Jr., Biopolymers 25, 205 (1986); W. H. Braunlin, Q. Xu, Biopolymers 32, 1703 (1992).

[8] M. Olvera de la Cruz, L. Belloni, M. Delsanti, J. P. Dalbiez, O. Spalla, M. Drifford, J. Chem. Phys. 103, 5781 (1995).

[9] E. Raspaud, I. Chaperon, A. Leforestier, F. Livolant, Biophys. J. 77, 1547 (1999); E. Raspaud, M. Olvera, M. O. de la Cruz, J.-L. Sikorav, F. Livolant, Biophys. J. 74, 381 (1998).

[10] J. F. Stilck, Y. Levin, J. J. Arenzon, J. Statistical Physics 106, 287 (2002).

[11] J. Pelta, F. Livolant, J.-L. Sikorav, J. Biological Chemistry 271, 5656 (1996); J. Pelta, D. Durand, J.Doucet, F. Livolant, Biophys. J 71, 48 (1996).

[12] M. Saminathan, T. Antony, A. Shirahata, L. H. Sigal, T. Thomas, T. J. Thomas, Biochemistry 38, 3821 (1999).

[13] F. J. Solis, M. O. De la Cruz, Eur. Phys. E. 4, 143 (2001). 
[14] F. J. Solis, J. Chem. Phys. 117, 9009 (2002).

[15] R. de Vries, Biophys. J. 80, 1186 (2001).

[16] Y. Murayama, Y. Sakamaki, M. Sano, Phys. Rev. Lett. 90, 018102 (2003).

[17] T. T. Nguyen, I. Rouzina, B. I. Shklovskii, J. Chem. Phys. 112, 2562 (2000); T. T. Nguyen, B. I. Shklovskii, J. Chem. Phys, 115, 7298 (2001).

[18] V. A. Bloomfield, Curr. Opin. Struct. Biol. 6, 334 (1996).

[19] Y. Burak, G. Ariel, D. Andelman, Biophys. J. 85, 2100 (2003).

[20] P. G. Arscott, A.-Z. Li, V. A. Bloomfield, Biopolymers 29, 619 (1990).

[21] F. J. Solis, M. O. De la Cruz, J. Chem. Phys. 112, 2030 (2000).

[22] V. S. Trubetskoy, J. A. Wolff, V. G. Budker, Biophys. J. 84, 1124 (2003).

[23] M. Tanaka, A. Yu. Grosberg, J. Chem. Phys. 115, 567 (2001).

[24] J. C. G. Montoro, J. L. F. Abascal, J. Chem. Phys. 103, 8273 (1995), J. Chem. Phys. 109, 6200 (1998); J. L. F. Abascal, J. C. G. Montoro, J. Chem. Phys. 114, 4277 (2001).

[25] E. Allahyarov, H. Löwen, G. Gompper, Phys. Rev. E. 68, 061903 (2003).

[26] E. Allahyarov, H. Löwen, Phys. Rev. E 62, 5542 (2000).

[27] E. Allahyarov, G. Gompper, H. Löwen, Phys. Rev. E. 69, 041904. (2004).

[28] E. Allahyarov, H. Löwen, G. Gompper, to appear in Euro. Phys. Letters (2004).

[29] G. S. Manning, Q. Rev. Biophys. 11, 179 (1978).

[30] H. H. Strey, V. A. Parsegian, R. Podgornik, Phys. Rev. E 59, 999 (1999).

[31] A. A. Kornyshev, S. Leikin, Phys. Rev. Lett. 84, 2537 (2000).

[32] D. Durand, J. Doucet, F. Livolant, J. Phys. II 2, 1769 (1992). 
[33] R. Podgornik, H. H. Strey, V. A. Parsegian, Curr. Op. Colloid Interface Science 3, 534 (1998).

[34] E. Allahyarov, I. D’Amico, H. Löwen, Phys. Rev. Lett. 81, 1334 (1998).

[35] J. D. Weeks, D. Chandler, H. C. Andersen, J. Chem. Phys. 54, 5237 (1971).

[36] R. Evans, in Liquids at Interfaces edited by J. Charvolin, J.F. Joanny, and J. Zinn-Justin (North-Holland, Amsterdam, 1990).

[37] A. Santos, M. Lopez de Haro, S. Bravo Yuste, J. Chem. Phys. 103, 4622 (1995).

[38] R. Goobes, O. Cohen, A. Minsly, Nucleic Acids Research 30, 2154 (2002).

[39] M. Deserno, F. Jimenes-Angeles, C. Holm, M. Lozada-Cassou, cond-mat 0104002 (2003).

[40] M. Deserno, F. Jimenez-Angeles, C. Holm, M. Lozada-Cassou, J. Phys. Chem. B 105, $10983(2001)$. 